\title{
The effect of continuous arterial infusion of Gabexate Mesilate (FOY-007) on experimental acute pancreatitis
}

\author{
Hirohiko Satoh, Masamitsu Harada, Seiki Tashiro, Tsutomu Shiroya*, Haruo Imawaka*, \\ and Koji Machii* \\ Department of Digestive and Pediatric Surgery, The University of Tokushima School of Medicine, \\ Tokushima, Japan ; and * Ono Pharmaceutical Co., Ltd., Osaka, Japan
}

\begin{abstract}
The effectiveness of continuous arterial infusion of Gabexate Mesilate (FOY- 007) on experimental acute pancreatitis was investigated. Acute necrotizing pancreatitis was induced by an injection of $10 \% \mathrm{Na}$-taurocholate $(1 \mathrm{ml} / \mathrm{kg})$ into the main pancreatic duct of mongrel dogs. Animals were divided into three groups; Group A: non-treated control, Group B : after the induction of pancreatitis, injected with FOY-007 intravenously $(5 \mathrm{mg} / \mathrm{kg} / \mathrm{hr})$, Group C : after the induction of pancreatitis, injected with FOY-007 via the celiac artery. The changes in the values of amylase and lipase in serum and ascites etc. were examined. A histological examination was done and the FOY-007 concentration of the pancreas was measured. In both groups B and C, the serum levels of amylase and lipase reached significantly to low levels compared with those in group $A$. The extents of pancreatic parenchyma necrosis in each group were 36.1, 25.3 and 19.5\%, respectively, and were significantly improved in group C. In addition, the FOY- 007 levels in pancreas specimens in the intraarterial infusion group exceeded those in the intravenous infusion group by 32 times. The results suggest that continuous FOY-007 arterial infusion therapy is useful as a local treatment for severe acute pancreatitis. J. Med. Invest. 51 : 186-193, August, 2004
\end{abstract}

Keywords : severe acute pancreatitis, Gabexate Mesilate, continuous arterial infusion therapy

\section{INTRODUCTION}

In severe acute pancreatitis the inflammation of the pancreas does not remain in the pancreas, local field and circumference. Due to a reaction of pancreatic enzymes and cytokines, which spread throughout the whole body, the inflammation affects the whole body. Then, neutral proteinase and active oxygen are discharged, with the activation of neutrophils. As a result, from the early crisis stage severe acute pancreatitis combines with multiple organ failure (MOF) and disseminated intravascular coagulation syndrome (DIC) (1). Therefore, a causal therapy for the pancreas local

Received for publication February 16, 2004 ; accepted March 1, 2004.

Address correspondence and reprint requests to Hirohiko Satoh, M.D., Department of Digestive and Pediatric Surgery, Institute of Health Biosciences, The University of Tokushima Graduate School, Kuramoto-cho, Tokushima770-8503, Japan and Fax:+81-88-8436385. field and a general treatment for MOF and DIC are required.

Gabexate mesilate (FOY-007) is a protease inhibitor with a broad spectrum inhibiting not only trypsin and kallikrein, but also plasmin, thrombin, factor Xa, elastase, complement 1 (C1), esterase and phospholipase A2 (2). Prophylactic application of FOY-007or Camostat mesilate (FOY-305) showed some beneficial effect in CDEinduced pancreatitis $(3,4)$, cerulein-induced panceratitis (5) and sodium taurocholate-induced panceratitis (3). The therapeutic effect of FOY-007 in acute panceratitis has been ascribed to its inhibition of pancreatic enzyme activity, like trypsin, thus preventing autodigestion (5).

The FOY-007 infusion method is generally an intravenous infusion method. In addition, there is the intraduodenal infusion method of Takasugi et al. (6) and the intraductal administration method of Wakayama et al .(7). Both studies reported that the spread of acute 
pancreatitis was prevented. However, there have been very few experimental reports on the intraarterial infusion method.

The purpose of the present experiment was to assess the therapeutic effect of continuous arterial infusion of FOY-007 in a lethal model of acute necrotizing pancreatitis in dogs.

\section{MATERIALS AND METHODS}

\section{Animals}

Adult mongrel dogs of both sexes, weighing between 10 and $20 \mathrm{~kg}$, were used throughout the study. They were kept under standard conditions $(12: 12 \mathrm{~h}$ lightdark cycles), and fed an ordinary laboratory diet (Clea, Japan) and water. All experiments were performed in animals after $12 \mathrm{~h}$ fasting with free access to water.

Canulation to the celiac artery and induction of acute necrotizing pancreatitis

All procedures were performed under sterile conditions. Adult mongrel dogs were anesthetized by intravenous injection of pentobarbital sodium $(50 \mathrm{mg} / \mathrm{kg}$, Abott Laboratory, North Chicago, III.,USA) followed by endotracheal intubation. They were maintained on controlled breathed $30 \%$ oxygen. The right femoral vein was cannulated with a 16 -gauge intravenous hyperalimentation catheter to infuse a lactated Ringer's solution at a rate of $10 \mathrm{ml} / \mathrm{kg} / \mathrm{h}$. The right femoral artery was cannulated with a 20 -gauge Teflon catheter to monitor arterial blood pressure for blood sampling. After an upper abdominal median incision, the left gastric artery was exposed and cut downwards, and a 3 Fr. Polyethylene catheter (ID, 1.0mm; Atom, Japan) was inserted in the retrogradation from the distance edge. The tip of the catheter was placed at the celiac artery. After Indigocarmine was injected from the catheter, we confirmed that the left and right lobes of the pancreas were stained. Acute necrotizing pancreatitis was induced by the injection of $10 \%$ sodium taurocholate $(1.0 \mathrm{ml} / \mathrm{kg}$ for $5 \mathrm{~min})$ to the main pancreatic duct.

\section{Experimental protocol}

Experimental animals were divided into three groups. Group A (non-treatment group, $\mathrm{n}=6$ ) : No drugs were given during the experiment. Group B : FOY-007 was given intravenously for $6 \mathrm{hrs}$ after the induction of pancreatitis $(5 \mathrm{mg} / \mathrm{kg} / \mathrm{hr}, \mathrm{n}=9)$. Group C: FOY-007 was given via the celiac artery for $6 \mathrm{hrs}$ after the induction of pancreatitis $(5 \mathrm{mg} / \mathrm{kg} / \mathrm{hr}, \mathrm{n}=9)$. The use of anesthesia during experimental procedures was consistent with the National Institutes of Health guidelines for the care and use of laboratory animals.

\section{Hemodynamic parameters}

A pressure transducer, Uniflow (Baxter Co.), was connected to the needle of a 20 gauge, which was inserted intraoperatively into the right femoral artery of each experimental animal. Using this transducer, the systolic arterial pressure (SAP, $\mathrm{mmHg}$ ) and diastolic arterial pressure (DAP, $\mathrm{mmHg}$ ) were measured in all experimental animals 6 hours after the operation. We then calculated the mean arterial pressure (MAP, $\mathrm{mmHg}$ ) in each animal. MAP was derived from the equation $\mathrm{DAP}+1 / 3 \times(\mathrm{SAP}-\mathrm{DAP})$.

\section{Serum and ascites samples}

Before, 1, 2, 4, and $6 \mathrm{~h}$ after the injection of $10 \%$ sodium taurocholate, blood was quickly withdrawn from the right femoral artery to measure amylase, lipase, alanine aminotransferase (ALT), lactic dehydrogenase $(\mathrm{LDH})$, calcium $(\mathrm{Ca})$, blood urea nitrogen $(\mathrm{BUN})$ and creatine (Cr). Also, 1, 2, 4, and $6 \mathrm{~h}$ after the injection of $10 \%$ sodium taurocholate, dogs were relaparotomized and ascites were quickly withdrawn from the Douglas pouch to measure amylase and lipase. Serum and ascites samples were stored at $-80^{\circ} \mathrm{C}$. Amylase was measured by enzymatic assay using an amylase assay kit (Pureauto amy-c, Daiichi Pure Chemicals CO. LTD), and lipase was measured by a spectrophotometric assay using a lipase kit (Liquitech Lipase color, Roche Diagnostic). ALT, LDH, Ca was measured using ALT, LDH and Ca assay kits (L-type Wako ALT, L-type Wako LDH, and Calcium E-H test Wako, respectively ; Wako Pharmaceutical Co., Ltd.). Serum BUN levels were measured, using Quick Auto II BUN (Sino Test, Co.), while serum creatinine levels were measured using an Ltype Wako Creatinine F (Wako Pharmaceutical Co., Ltd.).

\section{Histological examination}

Dogs were sacrificed $6 \mathrm{hrs}$ after an injection of $10 \%$ sodium taurocholate. The pancreas was removed and subsequently fixed with $10 \%$ formaldehyde. A slice was collected from the left lobe and from the main pancreatic duct bifurcation to a $2 \mathrm{~cm}$ interval, and hematoxylineosin staining was carried out on a total of 5 slices for histological examination. The ratio of pancreatic necrosis was measured with the image analysis software NIH image 1.61(NIH, Bethesda, Md., and USA). The area of pancreas substance was calculated for every slice, as well as the area of the parts that showed clear 
necrosis. The area ratio (the pancreatic necrosis area/ pancreas substance area), and mean value of pancreatic necrosis area/pancreas substance area ratio for all slices were also calculated. The mean values of each group were compared and examined.

\section{FOY-007 levels in pancreas specimens}

The pancreas was frozen in 3 dogs from group B and $\mathrm{C}$ in liquid nitrogen prior to sacrifice. Pancreatic tissue weighing $0.2 \mathrm{~g}$ was accurately collected from the left, center, and right end of the left lobe. The FOY007 concentrations were determined (ONO Pharmaceutical Co., Ltd). The FOY-007R concentrations were examined in terms of the mean value of each group.

\section{Statistical analysis}

Statistical analysis was performed using one-way analysis of variance and the Student's t test. Each result was expressed as the mean \pm SE. Statistical significance was set at $\mathrm{P}<0.05$.

\section{RESULTS}

\section{Hemodynamic parameters}

Before the experiment in group A, the systolic arterial pressure was $183 \pm 22 \mathrm{mmHg}$. However, the systolic arterial pressure 6 hours after the operation was significantly lower compared to that before the experiment. In group $B$, the systolic arterial pressure tended to be lower postoperatively : at 6 hours it was significantly low. In group C, 2 hours postoperatively it showed that a low value, but tended increase thereafter (Table 1). The mean arterial pressure showed the change was equal to the systolic arterial pressure (Table 1).
A

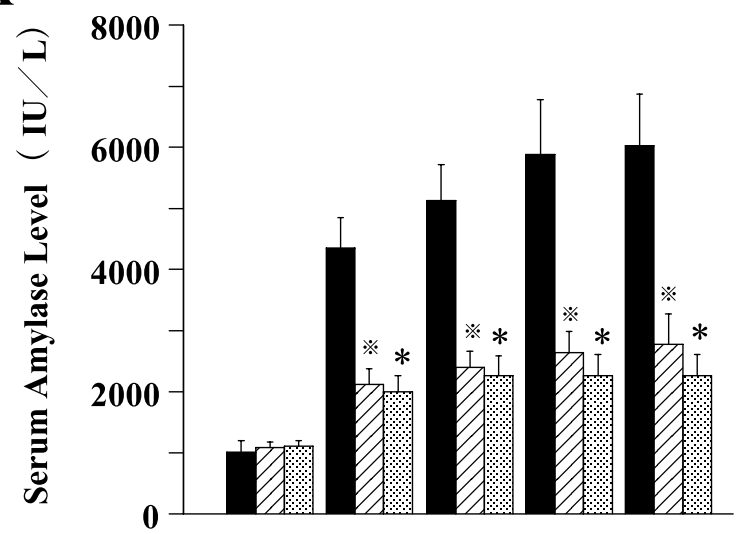

B

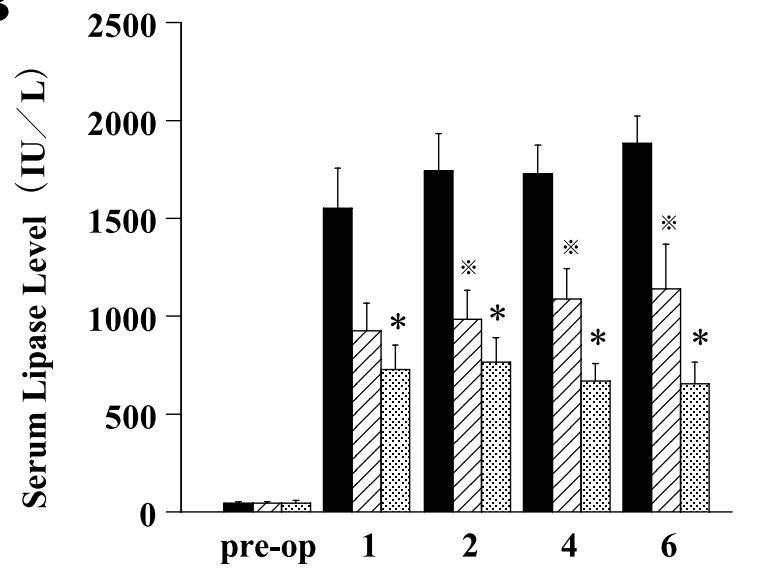

Time after induction of pancreatitis ( hrs. )

Fig 1. Serum amylase (A) and lipase (B) levels. $\mathbf{\square}$, Group A is the non-treatment group $(\mathrm{n}=6) ; \square \Delta$, Group B received FOY007 intravenously $(\mathrm{n}=6)$; $:$, Group $\mathrm{C}$ received FOY- 007 via the celiac artery $(\mathrm{n}=6)$, respectively. Symbols indicate the significant difference in each experimental group for time after induction of pancreatitis, as follows : $*(\mathrm{P}<0.01)$, Group A versus Group B. $*(\mathrm{P}<0.01)$, Group A versus Group C. Pre-OP, preoperation.

Table 1. Hemodynamic pressure parameters in each experimental group for time after induction of pancreatitis

\begin{tabular}{cccccc}
\hline Parameter & pre-op & $1 \mathrm{~h}$ & $2 \mathrm{~h}$ & $4 \mathrm{~h}$ & $6 \mathrm{~h}$ \\
\hline SAP(mmHg) & & & & & \\
Group A & $183 \pm 22$ & $137 \pm 40^{*}$ & $131 \pm 25^{*}$ & $143 \pm 20^{*}$ & $138 \pm 36^{*}$ \\
Group B & $173 \pm 53$ & $148 \pm 34$ & $141 \pm 29$ & $133 \pm 32$ & $127 \pm 33^{*}$ \\
Group C & $179 \pm 27$ & $145 \pm 23$ & $142 \pm 32^{*}$ & $154 \pm 34$ & $159 \pm 31$ \\
MAP(mmHg) & & & & \\
Group A & $136 \pm 13$ & $99 \pm 29^{*}$ & $98 \pm 21^{*}$ & $103 \pm 15^{*}$ & $103 \pm 23^{*}$ \\
Group B & $125 \pm 42$ & $115 \pm 27$ & $111 \pm 24$ & $102 \pm 25$ & $96 \pm 24$ \\
Group C & $132 \pm 14$ & $115 \pm 20$ & $111 \pm 24$ & $118 \pm 21$ & $121 \pm 23$ \\
\hline
\end{tabular}

Hemodynamic pressure parameters in each experimental group for time after induction of pancreatitis. Hemodynamic pressure parameters in each experimental group for time after induction of pancreatitis. Group A is the non-treatment group ( $\mathrm{n}=6)$, Group B received FOY-007 intravenously $(n=6)$, Group C received FOY-007 via the celiac artery $(n=6)$. Symbols indicate the significant difference of blood pressure in each experimental group for time after induction of pancreatitis, compared to that of preoparation. $*(\mathrm{P}<0.05)$, versus preoparation. ${ }^{*}(\mathrm{P}<0.01)$, versus preoparation. $S A P$, systolic arterial pressure. MAP, mean arterial pressure. 
A

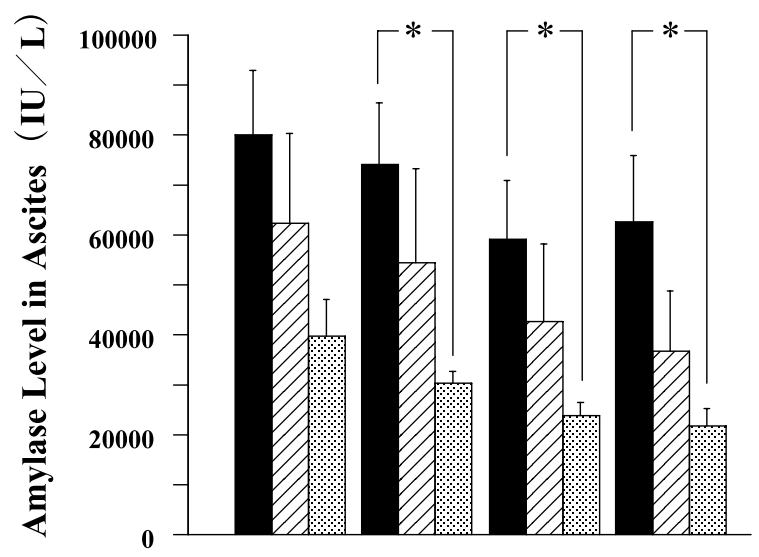

B

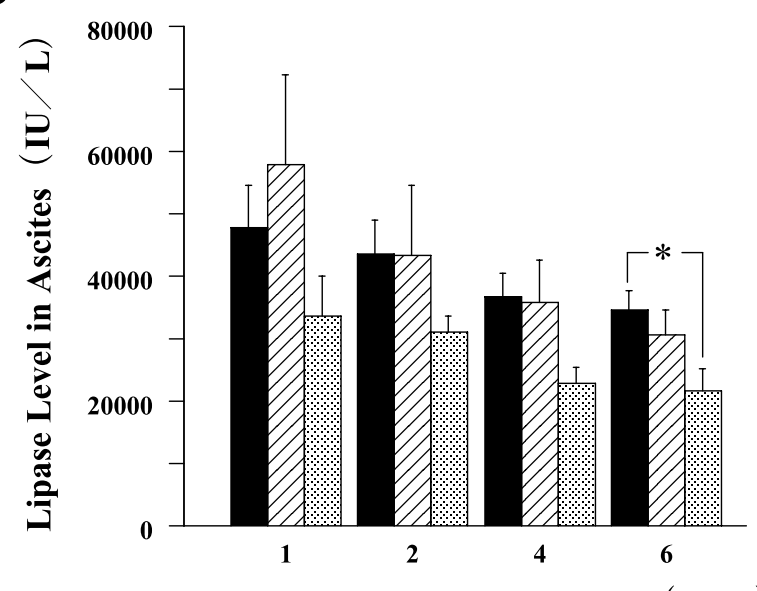

Time after induction of pancreatitis ( hrs. )

Fig 2. Amylase (A) and lipase (B) levels in ascites. $\square$, Group $A$ is the non-treatment group $(n=6) ; \square \Delta$, Group B received FOY007 intravenously $(\mathrm{n}=6)$; $: \mathrm{:}$, Group $\mathrm{C}$ received FOY-007 via celiac artery $(n=6)$, respectively. Symbols indicate the significant difference in each experimental group for time after induction of pancreatitis, as follows :* $(\mathrm{P}<0.05)$, Group A versus Group C.

\section{Serum amylase and lipase levels}

In group $\mathrm{B}$ and $\mathrm{C}$ amylase level in serum was significantly low compared with that in group $\mathrm{A}$ at all time points after induction of pancreatitis (Fig. 1A). In group $\mathrm{B}$ and $\mathrm{C}$ lipase level in serum was significantly lower compared with that in group $A$ at all time points after induction of pancreatitis (Fig. 1B).

\section{Amylase and lipase levels in ascites}

In group B, the amylase level in ascites was not significantly lower compared with that in group $\mathrm{A}$ at all time points after induction of pancreatitis, but in group $\mathrm{C}$ it was significantly low 2,4 , and $6 \mathrm{hrs}$ after induction of pancreatitis (Fig. 2A). Only the lipase level in ascites in group $\mathrm{C}$ was low compared with that in group $\mathrm{A}$ at all time points after induction of pancreatitis. Especially, it was significantly low $6 \mathrm{hrs}$ after induction of pancrea-
A

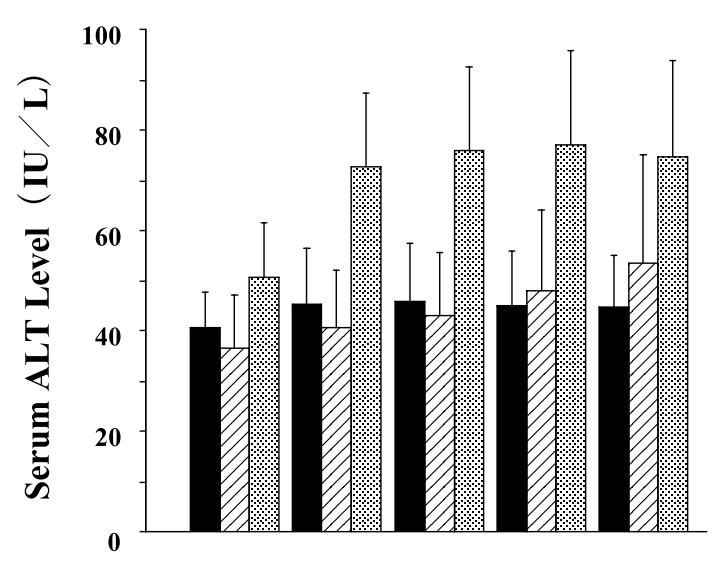

B

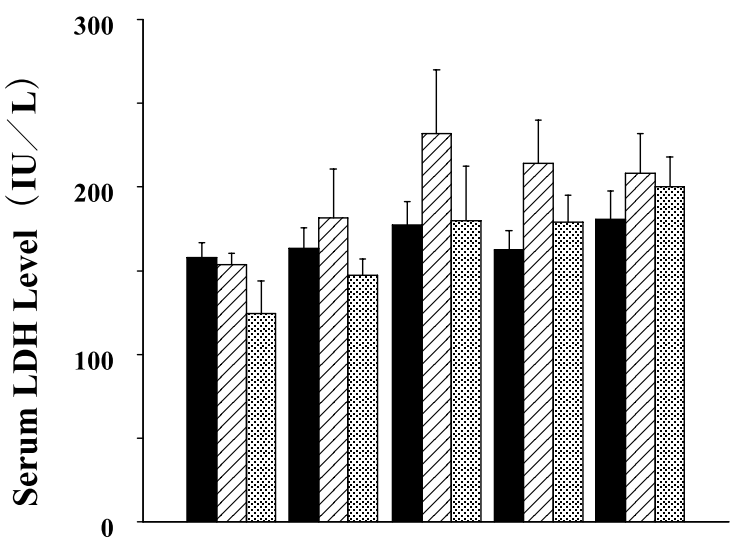

C

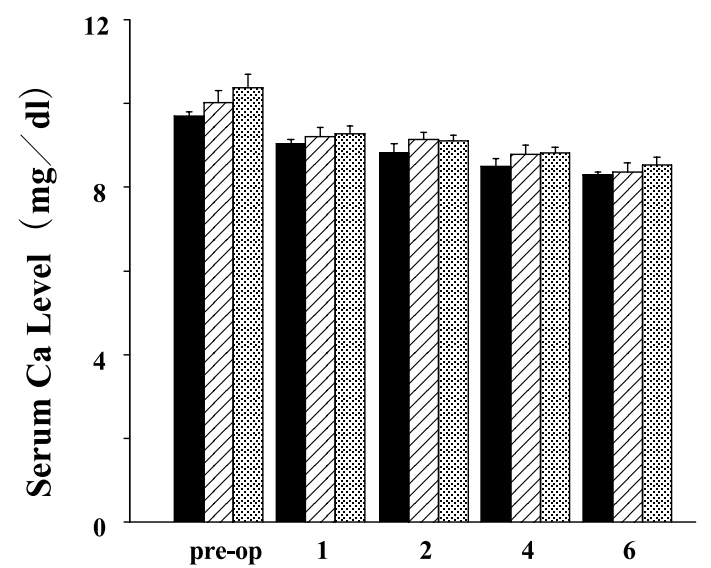

Time after induction of pancreatitis ( hrs. )

Fig 3. Serum ALT (A) LDH (B) and Ca (C) levels. $\mathbf{a}$, Group $A$ is the non-treatment group $(n=6) ; \square \Delta$, Group B received FOY007 intravenously $(n=6)$; : : , Group $C$ received FOY-007 via the celiac artery $(n=6)$, respectively. Pre-OP, preoperation.

titis (Fig. 2B).

\section{Serum ALT, LDH and Ca levels}

No significant differences in serum ALT, LDH and $\mathrm{Ca}$ levels were found in any group (Fig. 3A-C).

\section{Serum BUN and Cr levels}

In group $\mathrm{C}$, the BUN level was significantly low 1 , 

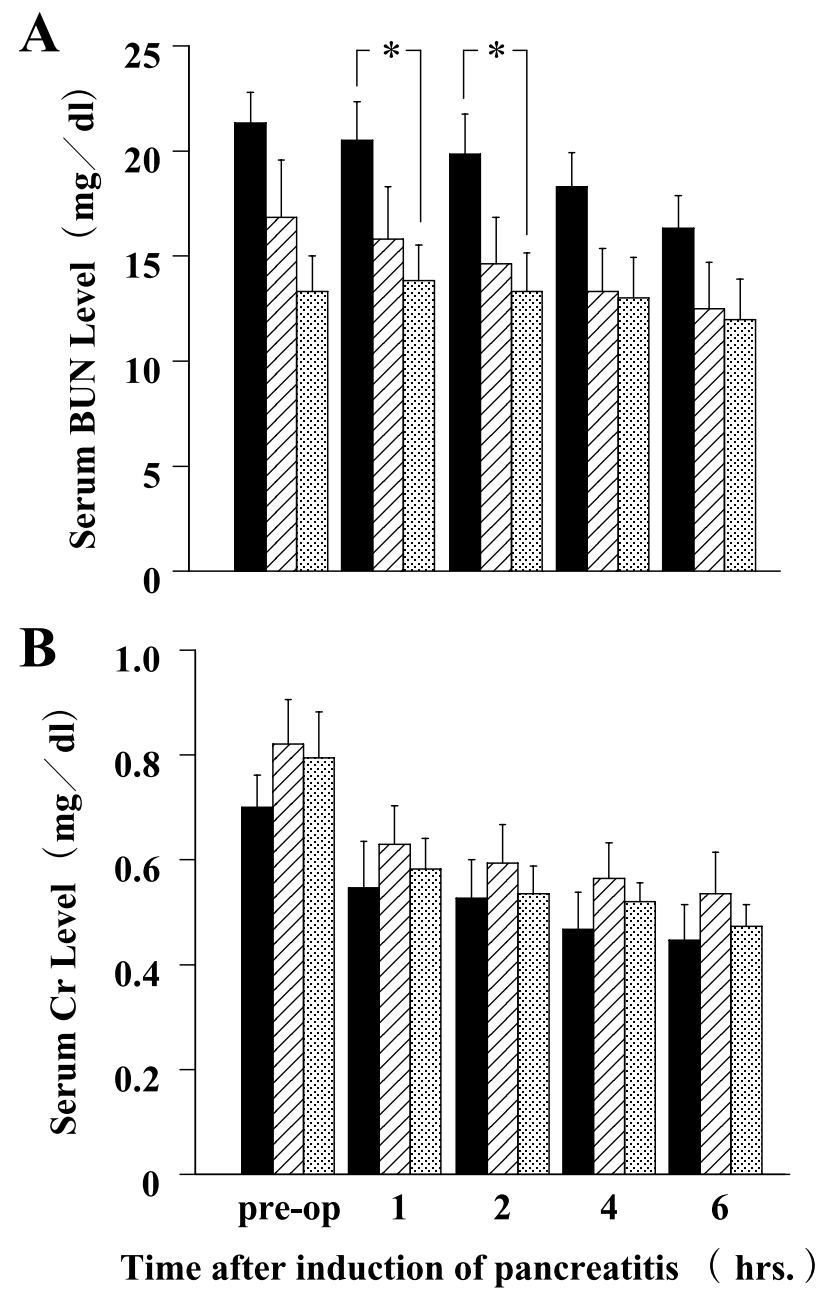

Fig 4. Serum BUN (A) and Cr (B) levels. $\square$, Group A is the nontreatment group $(\mathrm{n}=6) ; \square$, Group B received FOY-007 intravenously $(\mathrm{n}=6) ; \mathrm{:}$, Group C received FOY-007 via the celiac artery $(\mathrm{n}=6)$, respectively. Symbols indicate the significant difference in each experimental group for time after induction of pancreatitis, as follows. * $(\mathrm{P}<0.05)$, Group A versus Group C.

and $2 \mathrm{hrs}$ after induction of pancreatitis (Fig. 4A), but no significant differences in serum $\mathrm{Cr}$ levels were found in any group (Fig. 4B).

\section{Histological examination of the autopsy pancreas}

Falling and necrosis of the pancreatic substance and edema of the interstitial tissue were widely recognized in group A in the autopsy histological examination (Fig. 5A). In group B, the edema changes in the interstitial tissue remained, and the necrosis range was decreased compared with that in group A (Fig. 5B). In group $\mathrm{C}$, the edema change of the interstitial tissue remained slightly, and the necrosis range was slightly lower (Fig. 5C). The extents of pancreatic parenchyma necrosis in each group were $36.1 \pm 2.6,25.3 \pm 7.5$ and $19.5 \pm 2.3 \%$, respectively. Especially, in group $\mathrm{C}$ the extent of pancreatic parenchyma necrosis was significantly lower compared with that in group A (Fig. 6).
A

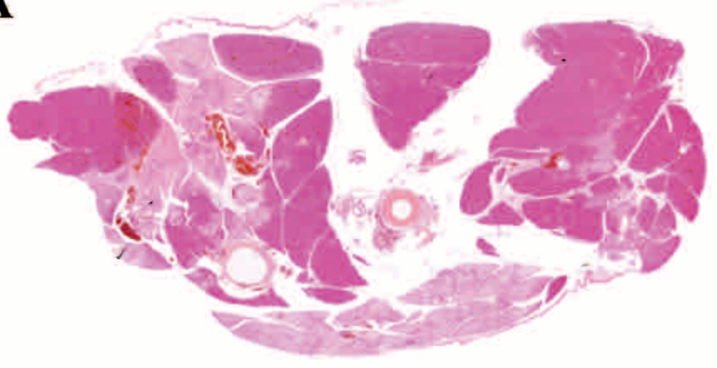

B

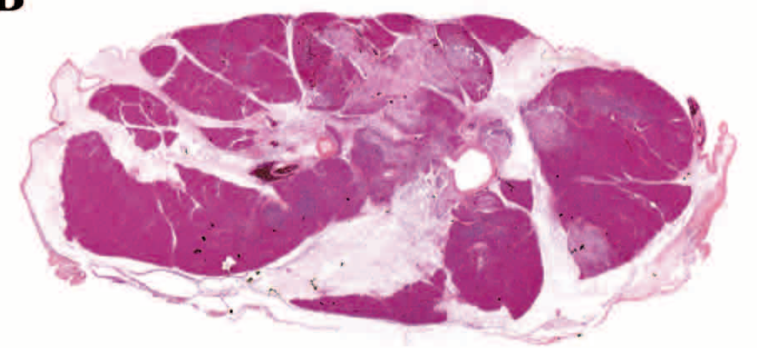

C

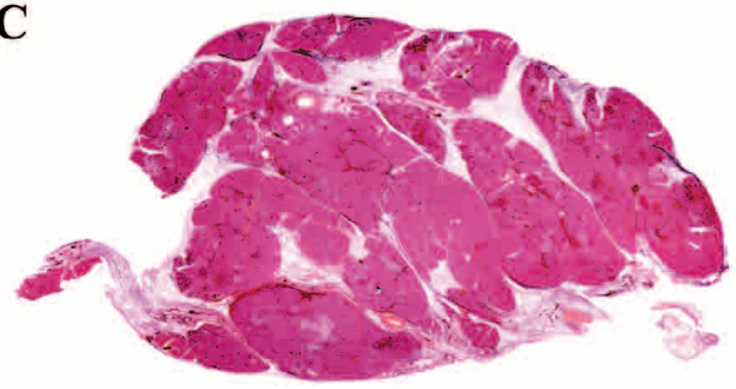

Fig 5. Histological findings of experimental animals. The findings of the pancreatic specimen, magnified 10 times (HE staining), taken by autopsy. A-C shows the findings of the specimen in group A-C.

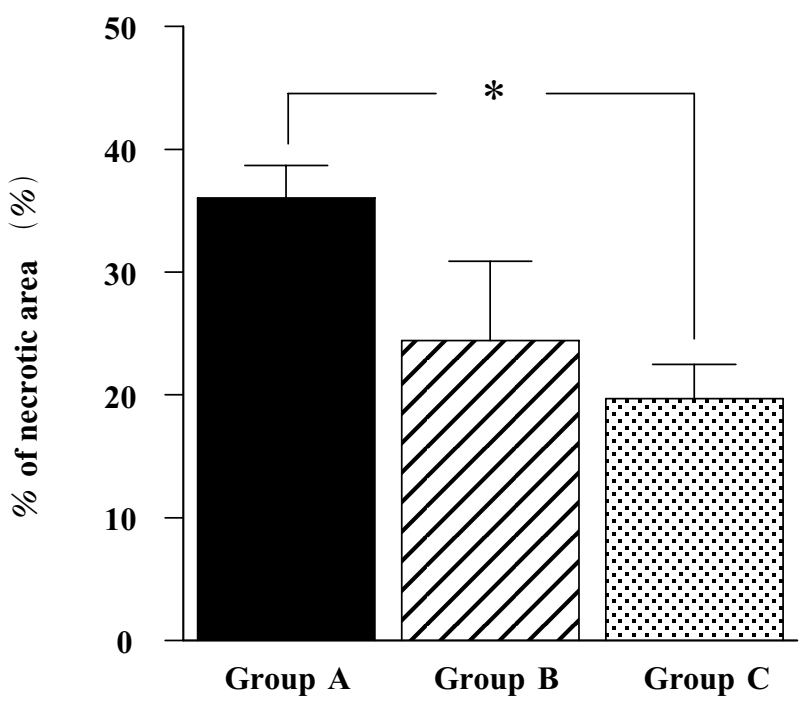

Fig 6. The ratio of pancreatic necrosis area/pancreatic substance area, taken by autopsy. $\square$, Group A is the non-treatment group $(\mathrm{n}=6) ; \square$, Group B received FOY-007 intravenously $(\mathrm{n}=6)$; : : Group C received FOY- 007 via the celiac artery $(n=6)$, respectively. Symbols indicate the significant difference in each experimental group 6 hours after induction of pancreatitis, as follows :* $(\mathrm{P}<$ 0.05), Group A versus Group C. 
A

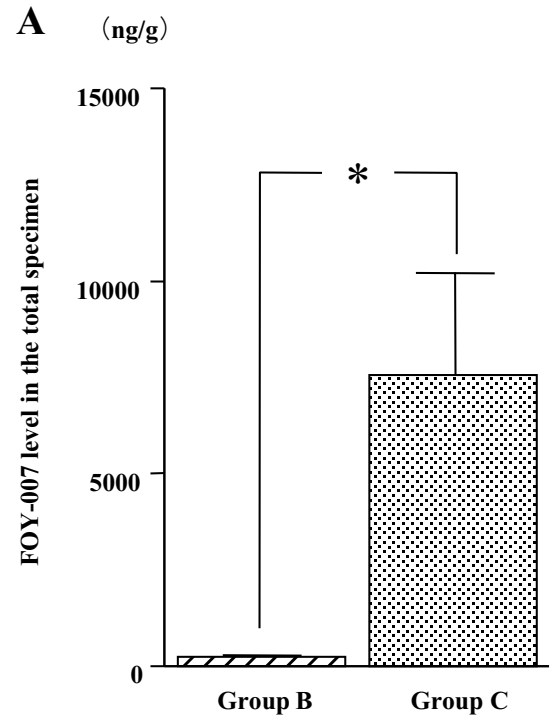

B

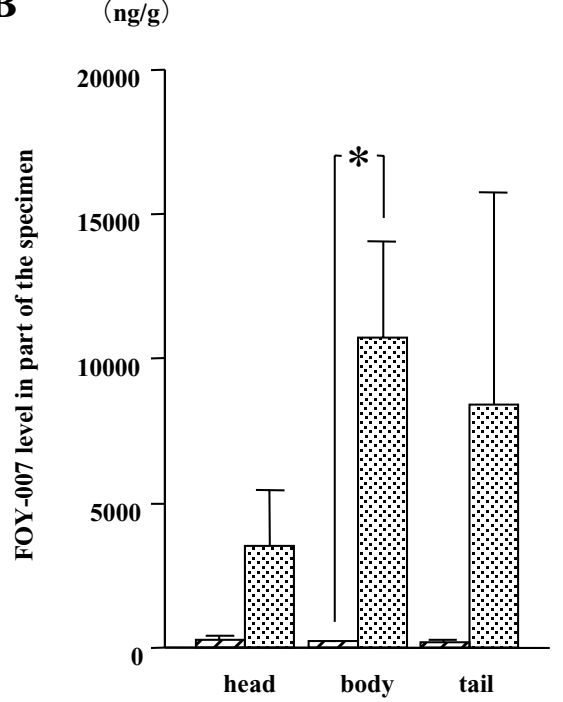

Fig 7. FOY-007 levels in the total pancreatic specimens (A) and part of the specimens (B). $\triangle A$, Group B received FOY-007 intravenously $(n=3)$; : : Group $C$ received FOY-007 via the celiac artery $(n=3)$, respectively. Symbols indicate the significant difference in both experimental groups 6 hours after induction of pancreatitis, as follows :* $(\mathrm{P}<0.05)$.

\section{FOY-007 levels in the pancreas specimens}

FOY-007 levels in the pancreas specimens in group B were $234.6 \pm 50.4 \mathrm{ng} / \mathrm{g}$, and $7562.1 \pm 2620.9 \mathrm{ng} / \mathrm{g}$ in group C. FOY-007 levels in the pancreas specimens in group $\mathrm{C}$ exceeded those in group $\mathrm{B}$ by 32 times (Fig. 7A). In comparison with the individual part of the pancreas, in group B, FOY-007 levels in the pancreatic head were $302.9 \pm 125.7 \mathrm{ng} / \mathrm{g}, 234.7 \pm 8.7 \mathrm{ng} / \mathrm{g}$ in the pancreatic body, and $166.1 \pm 99.5 \mathrm{ng} / \mathrm{g}$ in the pancreatic tail. In group C, FOY-007levels in the pancreatic head were $3528.6 \pm 1927.9 \mathrm{ng} / \mathrm{g}, 10735.7 \pm 3326.4 \mathrm{ng} / \mathrm{g}$ in the pancreatic body, and $8421.9 \pm 7356.0 \mathrm{ng} / \mathrm{g}$ in the pancreatic tail. In the pancreatic body, FOY-007 levels in group $\mathrm{C}$ were significantly higher than those in group B (Fig. 7B).

\section{DISCUSSION}

In severe acute pancreatitis, the inflammation of the pancreas local field spreads to not only the pancreas and pancreas circumference from the early stage, but also to the wholebody. As a result, the death rate is high, despite severe acute pancreatitis being a benign disease, because of DIC and MOF. It was reported that acute pancreatitis occurred in 45,000 people a year, while about 4,000 people died from complications in USA (8). In Japan, it was reported the death rate was $30 \%$ in a national investigation from 1982 to 1986, and $27 \%$ in 1996 . The therapy for severe acute pancreatitis was divided between topical treatment for the pancreas local field which was the primary cause of disease, and general treatment for important organ failure in the early stage, and multiple organ failure based on severe infectious disease in the later stage. We assumed the best way to prevent deterioration of acute pancreatitis was to control the inflammation in the pancreas local field in the early stage. There fore, we examined topical pancreas treatment. As a result, in the intraarterial injection group, systolic arterial pressure and mean arterial pressure were lowered immediately after the operation, and the improvement tendency was recognized from 2 postoperative hours. At 6 postoperative hours, it was improved without significance compared to the preoperative value. However, in the non-treatment and intravenous infusion groups, it lowered immediately after the operation, and the improvement tendency was not observed afterwards. This suggested that from the above result, FOY-007 continuous arterial infusion therapy improved early systemic circulation in the dynamic phase, and that it could prevent early organ failure.

In acute pancreatitis, trypsin is activated in zymogen granules of pancreatic acinus cells for various reasons $(9-11)$. Then, many varied deactivated pancreatic enzymes are activated by trypsin. As a result, the activated pancreatic enzymes deviate from the zymogen granules to interstitial tissue. This causes necrosis of the pancreatic acinus cells, edema of the interstitial tissue, failure of capillary and pancreatic ducts, and hemorrhaging necrosis of the pancreas circumference and retroperitoneum fat. In addition, the progress of this destruction induces the activation of macrophages, large cytokines, and the accumulation of neutrophils in the target organ. As a result, neutrophils which accumulate in target organ cause an organ disorder (12).

Historically, the intravenous injection of aprotinin, a protease inhibitor for acute pancreatitis, was widely performed. However, the effect of aprotinin was not adequately, because in the intravenous infusion of aprotinin, it was only slightly taken up in pancreas (13). The molecular weight of aprotinin is high at 6,512 . Therefore, the interference activity for trypsin which combines with $\alpha 2$-macroglobulin, an internal protease inhibitor, was very weak. It did not inhibit 
thrombin, Clr or Cls. Takeda et al. pointed out in 1987 since aprotinin of sufficient quantity in intravenous infusion did not reach to the pancreas, inflammation of the pancreas local field could not be controlled. The progress of pancreatitis could be suppressed by raising the aprotinin concentration in the pancreas. They devised continuous arterial infusion therapy with aprotinin and reported that there was no difference in pancreatic necrosis between the control group and the intravenous infusion group, while pancreatic necrosis was very slight in the intraarterial infusion group (14). In 1990, Kadokawa et al. found that the effect of continuous arterial infusion therapy using Nafamostat mesilate (FUT-175) had interference activity which was more powerful than aprotinin, its molecular weight is 540. They reported that in the intravenous infusion group, it insufficiently suppressed the progress of pancreatic necrosis, while the concentration of FUT-175 in the pancreatic tissues in the intraarterial infusion group was 5 times that in the intravenous infusion group. In addition, Trypsin Like Activity in the pancreas tissues was clearly lowered, so the progress of pancreatic necrosis was also suppressed (15). In this study, FOY-007 levels in the pancreas specimens in group $\mathrm{C}$ were 32 times those in group B. In comparison with individual parts of the pancreas, FOY- 007 levels in the pancreatic head were 12 times higher. In the pancreatic body, that in group $\mathrm{C}$ was 46 times that in group $\mathrm{B}$, which was significantly higher than that in group B. Also, in the pancreatic tail, that in the group $\mathrm{C}$ was 51 times that in group B. In the intravenous infusion group, in each part of the pancreas, FOY-007 levels were equal. In the meantime, in the intraarterial infusion group, of the body and tail of the pancreas and pancreas head, pancreas body was had an especially high value, because in the intravenous infusion group FOY- 007 flowed in the systemic circulation and only slightly flowed in the pancreas (16). Therefore, the intravenous infusion group showed lowvalues compared to the intraarterial infusion group which flowed directly in the pancreas. In the present experiment, the tip of intraarterial infusion catheter was in the celiac trunk. In short it was considered that FOY-007 levels in the pancreas head had decreased because of FOY-007 running off to the propria hepatic artery. The body and tail of the pancreas showed high values because they were near the tip of the catheter. Clinically, necrosis position and necrosis range of the pancreas are determined by diagnostic imaging. And the position and number of arterial catheters are examined for every case. The tip of the intraarterial infusion catheter is stored in the pancreatitis position, so it has a high value in the pancreas local field for the drug. This suggests that intraarterial infusion therapy can cure pancreatitis.

In the experimental examination of the infusion method of a protease inhibitor, Takasugi et al.(6) reported intraduodenal infusion of FOY-305 and FOY007 prevented acute pancreatitis in rats and dogs. Wakayama et al. (7) reported intraductal administration of FOY-007 prevented acute pancreatitis in dogs. The administration of a protease inhibitor to these duct systems has no rational when considered in terms of the mechanism of pancreatitis pathogenesis. However, a clinical method for administering a protease inhibitor in pancreatic ducts has not been established for severe acute pancreatitis. Therefore, only large intravenous or intraarterial injection allow us to place a protease inhibitor in the pancreas. At present, in clinics, continuous arterial infusion therapy using FUT175 widely used, and a marked effect has been reported. In the past, it was generally known that this medicine caused hyperkalemia due to an inhibitory effect of $\mathrm{Na}$ and K-ATPase activity of renal tubules. Recently, the secretion interference of Prostacin, a serine protease which appears in uriniferous tubule epithelial cells, was found to cause hyperkalemia ; in short, Prostacin was normally adjusting the electrolyte balance by activating the endothelial $\mathrm{Na}$ channel of distal renal tubules. However, FUT-175 inhibited the secretion of Prostacin, and suppressed the endothelial Na channel activity, and possibly causing hyperkalemia (17). The effectiveness of continuous arterial infusion of FOY007 that had an equal effect did not cause any electrolyte abnormality. The usefulness of FOY-007 for acute pancreatitis from the results of a multiple facility randomized comparison test in Europe was not confirmed (18). However, its usefulness in pancreatitis from the results of recent metanalysis was demonstrated (19). As a result, in the intraarterial infusion group, the amylase and lipase values in serum and ascites, and the pancreatic necrosis rate were significantly improved. A significant improvement in the intravenous infusion group in serum amylase and lipase values was noted. However, there was no significance for amylase, lipase values in ascites, or the pancreatic necrosis rate. Though the dose used this time was a quantity over $2 \mathrm{mg} / \mathrm{kg} / \mathrm{hr}$ for which the safety had been confirmed in the clinical analysis for DIC, the pancreatic necrosis depression effect due to the intravenous infusion was not sufficient. This is probably because in the intravenous infusion, a dilution effect due to blood occurs, and FOY-007 is rapidly hydrolyzed by esterase which is abundant in blood plasma (20). FOY-007 levels in specimens of the pancreas in the intraarterial infusion group exceeded 
those in the intravenous infusion group by 32 times, and the pancreatic necrosis was significantly suppressed. In addition, the increase in FOY-007 levels in the pancreas was obtained by the intraarterial infusion method, and an improvement in the pancreatic necrosis rate was expected.

In conclusion, continuous arterial infusion of FOY007 markedly reduce inflammation of the pancreas in an animal model of acute pancreatitis. We conclude that this method is clinically applicable in acute pancreatitis.

\section{REFERENCES}

1. Cavallini G, DI Francesco V, Bovo P: Antiprotease: new aspects of treatment. In : Buchler MW, Uhl W, Friess H, eds. Acute pancreatitis. Novel concepts in biology and therapy. Blackwell Science, Berlin, 1999, pp.299-305

2. Grozinger KH, Schrey A, Wabnitz RW : ProteinaseInhibition. Dr.C.Wolf \& Sohn, Munchen, 1981

3. Lankish PG, Pohl U, Goke B, OttoJ, WereszcynskaSiemiatkowska U, Grone H-J, Rahlf G : Effect of FOY-305(camostat) on severe acute pancreatitis in two animal models. Gastroenterology 96 : 193199, 1989

4. Niederau C, Liddle RA, Ferrell LD, Grendell JH : Benefical effects of cholecystokinin-receptor blockade and inhibition of proteolytic enzyme activity in experimental acute hemorrhagic pancreatitis in mice. Gastroenterology 96 : 193-199, 1989

5. Wisner JR, Renner IG, Grendell JH, Niederau C, Ferrell LD : Gabexate mesilate (FOY) protects against ceruletide-induced acute pancreatitis in the rat. Pancreas $2: 181-186,1987$

6. Takasugi S, Yonezawa H, Ikei N, Kanno T: Prevention of acute experimental pancreatitis in rats and dogs by intraduodenal infusion of a synthetic trypsin inhibitor. Digestion 24 : 36-41, 1982

7. Wakayama T, Itoh T, Shibayama K, Idezuki Y : Prevention of the spread of experimental acute pancreatitis by intraductal administration of a synthetic protease inhibitor in dogs. Am J Gastroenterol $84: 272-278,1989$

8. Bradley III EL, Warshaw AL: Pancreatic Abscess. The Pancreas : biology, pathobiology, and disease, 2nd ed, Go VLW, eds. Raven Press, New York, 1993, pp.643-663

9. Topazian M, Gorelick FS. Acute pancreatitis. In: Yamada T, Alpers DH, Laine L, eds. Textbook of Gastroenterology, Third Edition, Lippincott Wil- liams \& Wilkins, Philadelphia, 1999, pp.2121-2150

10. Steer ML: Pathogenesis of acute pancreatitis. Digestion 58 (Suppl 1) : 46-49, 1997

11. Leach SD, Modlin IM, Scheele GA, Gorelick FS : Intracellular activation of digestive zymogens in rat pancreatic acini. Stimulation by high dose of cholecystokinin. J Clin Invest 87 : 362-366, 1991

12. Ogawa M:Mechanisms of the development of organ failure following surgical insult : the'second attack' theory. Clin Intens Care $7: 1-5,1996$

13. Kaller H, Patzschke K, Wegner LA: Pharmacokinetic observations following intravenous administration of radioactively labelled aprotinin in volunteers. Eur J Drug Metabol Pharmacokinet 3 : 79-85, 1965

14. Takeda K, Matsuno S, Sunamura M, Miyagawa K, Sato T: Effect of continuous intraarteial infusion trypsin inhibitor on acute experimental pancreatitis in dogs. J Jpn Panc Soc 2 (in Japanese) :151158,1987

15. Kakugawa Y, Takeda K, Sunamura M, Kawaguchi S, Kobari M, Matsuno S : Effect of continuous arterial infusion of protease inhibitor on experimental acute pancreatitis induced by closed duodenal loop obstraction. Jpn J Gastroenterol 87 (in Japanese) : 1444-1450, 1990

16. Masayasu S, Tsumoru M, Hidetoshi Y:Studies on Absorption, Distribution, Excretion and Metabolism of Ethyl p-(6-guanidinohexanoyloxy) benzoate methanesulfonate. Pharmacometrics $9: 733-742,1975$

17. Kenichirou K, Kimio T:Secretion mechanism of the prostaglandin in the renal uriniferous tubule and examination of the clinical significance. The Kumamoto Univ. Medical School Third Medicine : Sixth disease state and protease in the treatment and inhibitor workshop. 2001 August 34 (in japanese).

18. Buchler M, Malferthiner P, Uhl W, Scholmerich J, Stockmann F, Adler G, Gaus W, Rolle K, Beger HG : Gabexate mesilate in human Acute pancreatitis. Gastroenterology 104 : 1165-1170, 1993

19. Messori A, Rampazzo R, Scroccaro G, Olivato R, Bassi C, Falconi M, Pederzoli P, Martini N : Effectiveness of gabexate mesilate in acute pancreatitis :a meta-analysis. Dig Dis Sci 40 : 734-738, 1995

20. Nishijima MK, Takezawa J, Taenaka N, Shimada Y, Yoshiya I : Application of HPLC measurement of plasma concentration of gabexate mesilate. Thromb Res 31 : 279-284, 1983 